\title{
La crisis económica de ecuador y su incidencia en el servicio al cliente
}

\author{
Ing. Christopher Carchipulla Alvarado \\ Instituto Tecnológico Formación
}

christopher.carchipulla@formacion.edu.ec

Palabras claves: servicio, cliente, crisis.

Keywords: service, clients, crisis.

\author{
Lic. María José Menéndez Ledesma \\ Instituto Tecnológico de Formación \\ maria.menendez1@formacion.edu.ec
}

Recibido: 13 de mayo de 2017

Aceptado: 10 de junio de 2017

\section{RESUMEN}

El mundo actual está lleno de cambios y la competencia empresarial cada vez es más intensa, los países en vías de desarrollo empiezan a despertar ganando mercado con precios competitivos y es necesario que Ecuador logre un nivel de competitividad óptimo para ganar espacio en cada vez más industrias, pero el cambio empieza desde la microempresa. Es preciso erradicar la cultura hacia el cliente basado en la mediocridad y maltrato, pulir el servicio y poner al cliente en el puesto que siempre debió ser suyo, el protagonismo. Ciertas empresas exigen al colaborador un buen trato al cliente, sin embargo, ellos mismos no lo hacen con el colaborador; es importante que para poder exigir al empleado una predisposición hacia el servicio, primero se le debe brindar un buen trato al empleado y un clima laboral favorable para el crecimiento profesional de su talento. Si los objetivos profesionales del colaborador podrían ser alineados a la misión y visión empresarial se tendrían una gran participación activa y un verdadero núcleo empresarial, elaborando una cadena de valor integrada con el único fin, la satisfacción del cliente.

\begin{abstract}
The current world is full of changes and business competition is becoming more intense; developing countries are beginning to grow in markets with strategies like competitive prices; it's necessary that Ecuador achieve an optimal level of competitiveness and gain space in more industries, but change starts from microenterprise, the culture of being without a service should be eradicated to the client based on mediocrity and abuse, polish the service and put the client in the position that should always be, the protagonism. Certain companies require the employee a good service to the client, however, they do not themselves with the employee; It is important that in order to be able to require the employee to be predisposed to the service, they must started by given a good treatment to the employee and a favorable working environment for the professional growth of his talent. If the professional goals of the employee could be aligned to the mission and business vision, they would have a great active participation and a true business join, elaborating an integrated value chain with the only purpose, customer satisfaction.
\end{abstract}




\section{INTRODUCCIÓN}

Diversos factores son los que han llevado al Ecuador a una crisis financiera nacional, encontrándose entre ellos: el terremoto que ocurrió el pasado 16 de abril del 2016 y que dejó como resultados pérdidas humanas y económicas. De la misma forma se vio afectada la industria del turismo con un descenso considerable en visitantes tanto nacionales y extranjeros. Asimismo, el precio del petróleo disminuyó considerablemente y afectó el presupuesto general del estado, a todo ello se suma la creación de nuevas políticas gubernamentales como el aumento y creación de impuestos que dieron como resultado el alto índice de desempleo y subempleo que, directamente, afectó la calidad de vida de los ecuatorianos.

De acuerdo a todo lo citado anteriormente, hoy día se puede evidenciar en las diferentes empresas tanto públicas y privadas del país, la mala atención hacia el cliente, originado a su vez por la falta de competencias emocionales en los trabajadores, definidas como: "conjunto de conocimientos, destrezas y aptitudes necesarias para ejercer una profesión, resolver problemas profesionales de forma autónoma y flexible y ser capaz de colaborar con el entorno profesional y en la organización del trabajo". (Bunk, 1994). Lo anterior trajo como resultado que la mayor parte del personal de las empresas ofrezca una mala atención hacia el cliente ya que hoy en día, gran parte de las empresas, por el afán de obtener rentabilidad hacen del mercado ecuatoriano poco competitivo y comprometido hacia el objetivo, meta, visión y misión de las empresas.

En las organizaciones contemporáneas hay una tendencia cada más creciente a una cultura manejada por los colaboradores denominada "Yoismo", que se convierte en una inadecuada herramienta para el manejo de la buena atención. Este virus, síndrome u obsesión por nada más que sí mismo ha generado una especie de "ceguera" en el ámbito emocional, mental y social que se manifiesta en todo aquel que se niega a abrir la mente, a ir más allá de su cada vez más reducido espacio vital.

Ejercitar la mente con un mínimo esfuerzo ayuda a que las cosas fluyan más rápidamente y a prevenir la ceguera para no pasar por este mundo terrenal como los caballos en competencia con las anteojeras que les limitan la visión. Es por ello que se evidencia la necesidad de erradicar la cultura del yoismo, con la finalidad de hacer que la empresa trabaje como un núcleo por un bien común, beneficiando al trabajador y la organización.

En relación a lo expuesto anteriormente, el objetivo del presente artículo consiste en realizar una revisión documental con el fin de analizar en qué medida las amenazas externas han incidido negativamente en la atención que brindan las organizaciones. 


\section{DESARROLLO}

El servicio al cliente es el servicio o atención que una empresa o negocio brinda a sus clientes al momento de atender sus consultas, pedidos o reclamos, venderle un producto o entregarle el mismo. Para entender mejor su concepto veamos a continuación los factores que intervienen en el servicio al cliente:

- Amabilidad: hace referencia al trato amable, cortés y servicial. Se da, por ejemplo, cuando los trabajadores saludan al cliente con una sonrisa sincera, cuando le hacen saber que están para servirlo, cuando le hacen sentir que están genuinamente interesados en satisfacerlo antes que en venderle.

- $\quad$ Atención personalizada: es la atención directa o personal que toma en cuenta las necesidades, gustos y preferencias particulares del cliente. Se da, por ejemplo, cuando un mismo trabajador atiende a un cliente durante todo el proceso de compra, cuando se le brinda al cliente un producto diseñado especialmente de acuerdo a sus necesidades, gustos y preferencias particulares, etc.

- $\quad$ Rapidez en la atención: es la rapidez con la que se le toman los pedidos al cliente, se le entrega su producto, o se le atienden sus consultas o reclamos. Se da, por ejemplo, cuando se cuenta con procesos simples y eficientes, cuando se cuenta con un número suficiente de personal, cuando se le capacita al personal para que brinden una rápida atención, etc.

- $\quad$ Ambiente agradable: es un ambiente acogedor en donde el cliente se siente a gusto. Se da, por ejemplo, cuando los trabajadores le dan al cliente un trato amable y amigable, cuando el local del negocio cuenta con una buena decoración, una iluminación adecuada, una música agradable, etc.

- $\quad$ Comodidad: hace referencia a la comodidad que se le brinda al cliente cuando visita el local. Se da, por ejemplo, cuando el local cuenta con espacios lo suficientemente amplios como para que el cliente se sienta a gusto, sillas o sillones cómodos, mesas amplias, estacionamiento vehicular, un lugar en donde pueda guardadas sus pertenencias, etc.

- $\quad$ Seguridad: se refiere a la seguridad que existe en el local y que, por tanto, se le da al cliente al momento de visitarlo. Se da, por ejemplo, cuando se cuenta con suficiente personal de seguridad, cuando se tienen claramente marcadas las zonas de seguridad, cuando se tienen claramente señalizadas las vías de escape, cuando se cuenta con botiquines médicos, etc.

- Higiene: limpieza o aseo que hay en el local o en los trabajadores. Se da, por ejemplo, cuando los baños del local se encuentran siempre limpios, cuando no hay papeles en el piso, cuando los trabajadores están bien aseados, con el uniforme o la vestimenta impecable y las uñas recortadas, etc.

Una empresa o negocio brinda un buen servicio al cliente cuando ha trabajado en varios de estos factores; por ejemplo, cuando trata a sus clientes con amabilidad, les da un trato personalizado, los atiende con rapidez, les ofrece un ambiente agradable, y los hace sentir cómodos y seguros. 
Durante los últimos meses, Ecuador ha pasado por varios momentos de crisis, los que han repercutido de forma directa en las diferentes empresas teniendo como punto en común, la falta de una cultura corporativa enfocada hacia el cliente; percibiendo a la crisis económica como un vaso medio lleno en vez de verlo como un vaso medio vacío.

Diversas son las herramientas que existen para mejorar las técnicas de servicio al cliente y brindar la oportunidad a las empresas de poder reinventar, con la intención que puedan llegar a ser pioneros en muchos ámbitos, entre los cuales se ha decidido estudiar básicamente el servicio al cliente, evaluando problemas, pero más aun ofreciendo soluciones.

Dentro de las insuficiencias más repetitivas encontradas en las organizaciones es la falta de interés del personal al momento en que un cliente se va sin realizar ninguna transacción, debido a la existencia de muchos clientes en el mercado; por consiguiente, la visión cambia ahora, en tiempos de crisis donde es cuando los clientes escasean. Ahora los vendedores en centros comerciales pasaron de ser reactivos a proactivos, es decir salir a buscar clientes, se cuida mucho de la fidelización y del servicio post-venta.

Definitivamente el servicio al cliente es mucho más profesional ahora, es decir el servicio al cliente ha mejorado sin embargo la atención al cliente aún no, pero para que la atención sea dirigida hacia el cliente, primero se debe tener la atención dirigida hacia el colaborador. Es el colaborador quien tiene contacto directo con el cliente y es el que va a desarrollar la magia directamente hacia el cliente.

Para ello, es muy importante resaltar la diferencia entre atención al cliente y servicio al cliente. En su libro Atención al Cliente, Juan Carlos Giraldo, señala:

La atención al cliente es la relación interpersonal amable y cordial que les brindamos a las personas con las que se entra en contacto para ofrecerle nuestros productos o servicios. El servicio al cliente incluye, adicionalmente a la atención, otros aspectos no menos importantes que el cliente evalúa a la hora de manifestar su satisfacción o insatisfacción. (Giraldo, 2011).

Por lo tanto, se necesita crear una relación más allá de la venta tradicional, basada en la fidelización del cliente, creando la relación cliente-servidor. Por otra parte, según Tigani (2006) el servicio, después de todo es: "la única razón de ser de cualquier organización" (pág. 3), lo cual confirma la premisa que la atención al cliente es importante e infaltable en la sociedad ecuatoriana.

Sin embargo, el problema actual de las empresas radica en la predisposición del colaborador hacia el cliente, debido a que la empresa está llena de burocracia que detiene la iniciativa del mismo. Estos factores junto a una mala relación entre em- 
pleado-empleador disminuirán el rendimiento del colaborador frente a su cliente, por tal motivo es importante encontrar la solución de forma radical, es decir buscar la raíz del problema, para lo cual es importante revisar la teoría de McGregor, donde plantea las Teorías "X y Y".

McGregor (1994) globaliza los dos tipos de pensamiento gerenciales hacia el staff netamente diferenciados por las ideologías de los líderes. En La teoría X se encuentran los empleados que trabajan por que no les queda de otra, convirtiendo al trabajador en un ser humano ordinario. Debido a esta tendencia humana a rehuir al trabajo, la mayor parte de las personas tienen que ser obligadas, controladas y dirigidas, y amenazadas con castigos para que desarrollen el esfuerzo para la realización de los objetivos de la organización.(p. 34).

Para lo cual, bajo un régimen marcado en el castigo donde hay que arrear al empleado, no es algo factible para desarrollar un servicio al cliente óptimo, debido a que el colaborador nunca va a estar comprometido con el cliente, recibiendo ese trato por parte de la empresa.

En la teoría "Y" sin embargo se expresa la necesidad del colaborador de asumir los objetivos vinculándolos con su crecimiento profesional, además se siente la motivación de perfeccionarse, y buscan las responsabilidades sin necesidad de amenazas o coerciones.

Asimismo, Chiavenato (2009) plantea una solución denominada "ganar-ganar" donde las metas profesionales del colaborador están ligadas a los objetivos de la organización; el colaborador es el talento y por lo tanto un talento debe de ser respetado, dirigido, y por supuesto cuidado. De esta manera no sólo gana la empresa, gana el colaborador y aún más importante gana el cliente.

Finalmente, se coincide con Neuman (2006) al mencionar los principales motivos por los que se pierden los clientes:

1.- carencia de una cultura de un servicio

2.- relaciones directas e indirectas con la competencia

3.- carencia de competitividad en los precios

4.- inestabilidad en la calidad de los productos

5.- movilidad de los clientes

Para establecer la calidad de los servicios en las empresas aparecen entoces las normas ISO 9000:2000 que detallan claramente las reglas que evalúan, considerando puntos claves como el liderazgo, que va mucho más allá a la atención al cliente, busca la raíz de la atención, siempre direccionándose hacia la meta final, la plena satisfacción al cliente. 
De acuerdo a las normas ISO el servicio se lo mide de la siguiente manera:

- Prontitud en el servicio.

- Puntualidad en la entrega.

- Cumplimiento en el tiempo del ciclo del servicio.

- Exactitud en el cumplimiento del servicio.

- Relación beneficio-costo.

- Personal calificado para el servicio adquirido.

- Cumplimiento de los plazos acordados.

- Amabilidad y buen trato en la presentación del servicio.

- Servicio asociado con lo pactado.

Cumpliendo todos estos estándares de calidad, es cuando la empresa obtendrá un cliente satisfecho.Asimismo, es importante resaltar que actualmente las empresas se encuentran en la era de la comunicación y del Internet, donde los clientes desean estar cada vez más informados sobre sus productos convirtiendo de esta forma al internet como un canal más de atención al cliente, motivo por el cual es necesario que las empresas contraten a un personal específicamente para que atienda los diferentes requerimientos y puedan de esta forma sacar un verdadero provecho a las redes sociales.

Un community manager no puede ser una persona que se dedica a poner "me gusta" y "compatir" en todos lados, debe ser un completo estratega, con carisma y facilidad para empatizar y construir relaciones. Con la libertad y responsabilidad suficientes para tomar decisiones de cierta importancia en la empresa que representa, ya que de lo contrario los clientes virtuales no harán otra cosa que perder el tiempo.

Es por ello que el servicio al cliente no se trata solamente de ofrecer los mejores productos, también es necesario que el ejecutivo de ventas y personal en general sea amable y se preocupe por las necesidades del cliente y por cómo satisfacerlas correctamente. Apareciendo de esta forma el servicio post venta, donde muchas de las empresas ecuatorianas aún no lo implementan.

Por eso, la primera regla que debe seguir el empresario o dueño del negocio para fidelizar a sus clientes es conocerlos bien. Saber todas sus necesidades relacionadas con el bien o servicio que se le puedaproporcionar.

Según Actions DATA, el primer paso para fidelizar a un cliente es entenderlo, yaque en tiempos de crisis las necesidades de las personas cambian y resulta de vital importancia entender sus motivaciones. La gestión y la explotación eficaz de las bases de datos (ya sean de clientes actuales, de suscripciones, de clientes potenciales, de asociados, etc.), que son uno de los principales valores de la empresa, permite hacer propuestas innovadoras y diferenciadas. 
Cuanto mejor se conozca a los actuales y potenciales clientes más posibilidades se tienen de adaptarse al mercado, y a los cambios que en éste se producen.

El segundo paso es invertir de forma inteligente, estudiando bien qué productos, mensajes y medios elegir. Invertir, por ejemplo, en CRM (Customer Relationship Management) ayuda a mejorar las ventas cruzadas o el upselling (vender productos de más valor), así como a incrementar la satisfacción del usuario y las ventas en general.

También es fundamental seguir el rastro del consumidor, analizar la información que deja a través de la web o mediante una encuesta y conocer el consumo que hace sobre los productos y servicios o la respuesta que ha tenido frente a las campañas realizadas.

Es importante recalcar que de acuerdo al informe anual de Accenture, Encuesta sobre el Pulso Global del Consumidor de 2013, una investigación sobre el impacto de la atención al cliente, revela que el $66 \%$ de los consumidores se cambian a la competencia debido a un pobre servicio al cliente. Pero, además, el $81 \%$ de los consumidores afirma que la empresa de la cual son clientes podría haber hecho algo más para evitar que ellos efectuasen tal cambio.

De la misma forma es importante resaltar que existen diferentes herramientas que se pueden utilizar para mejorar el servicio al cliente en una empresa, más aún en tiempo de crisis donde la competencia, cada vez es más dura, se debe evaluar los procesos, con la finalidad de ponerlos a prueba y buscando mejoras, para crear la fidelización y de esta forma el cliente prefiera y recomiende a la empresa.

Las encuestas son una de las herramientas que se pueden utilizar para mejorar el servicio al cliente, desarrollando preguntas cortas y adecuadas de lo que se desea saber para evitar molestias al cliente y así obtener la información necesaria.Los buzones de sugerencias son también muy importantes pues a través de ellos el cliente sin temor a represalias puede exponer quejas o críticas constructivas sin miedo ni vergüenza de decirlas frente al empleado.

De igual manera, la entrevista personalizada al cliente constituye una herramienta importante, además de ser una herramienta eficaz, es una técnica de obtención de información directa y primaria, cuya principal aplicación es analizar desde una perspectiva descriptiva y evaluadora el trabajo de los vendedores.

El buen servicio al cliente debe estar presente en todos los aspectos de un negocio en donde exista alguna interacción con el cliente, desde el saludo del personal de seguridad que está en la puerta del local, hasta la llamada contestada por la secretaria. Con el fin de lograr lo anterior, es necesario capacitar y motivar permanentemente al personal para que brinde un buen servicio al cliente, no solo aquellos trabajadores que tengan contacto frecuente con el cliente, sino a todos los que en algún momento 
puedan llegar a tenerlo, desde el encargado de la limpieza hasta el gerente general. Asimismo, el buen servicio al cliente no solo debe darse durante el proceso de venta, sino también después de la venta, pues el servicio post venta es una de las mejores formas de generar lealtad hacia la empresa y conseguir la fidelidad de los clientes y un cliente satisfecho es la mejor publicidad que una empresa puedetener.

Cuando se atraviesa por tiempos difíciles, es época en que las empresas se conviertan en creativas e ingeniosas, evitando de cualquier manera permanecer en un constante desánimo y frialdad.

El personal de las diferentes empresas deben mostrar siempre la mejor sonrisa, actitud, atender sin excusas, prestar atención ante los diferentes requerimientos de los clientes. Si bien es cierto el servicio no requiere de un gran esfuerzo, pero si requiere de la mejor sonrisa, el mejor gesto y la mejor actitud, lo cual marcará la diferencia.

\section{CONCLUSIONES}

El servicio al cliente debe estar presente en todos los aspectos del negocio en donde haya alguna interacción con el cliente, desde el saludo del personal de seguridad que está en la puerta del local, hasta la llamada contestada por la secretaria. Para lo cual es necesario capacitar y motivar permanentemente al personal para que brinde un buen servicio al cliente, no solo a aquellos trabajadores que tengan contacto frecuente con el cliente, sino a todos los que en algún momento puedan llegar a tenerlo, desde el encargado de la limpieza hasta el gerente general. Asimismo, el buen servicio al cliente no solo debe darse durante el proceso de venta, sino también una vez que esta se haya concretado.

En la actualidad, existe una gran competencia entre las empresas, debido a la existencia de productos similares, siendo la atención al cliente el punto diferenciador entre ellas, es por ello importante preguntar si: ¿el cliente es feliz con el servicio al cliente que recibe?

Es importante dar un buen servicio por la sencilla razón de que la está dando usted, si lo ve superior es un premio de consolación, las personas reconocen a un servidor como un individuo ilustre de la sociedad. El dinero es un subproducto de ser felices realizando una actividad, descubrir el servidor que cada persona lleva dentro. Hoy en crisis se puede ver que se han hecho muchas cosas mal, pero siempre se tiene una nueva oportunidad para poder recomenzar, poder trabajar en equipo y construir una sociedad diferente, desarrollar una nueva visión hacia el futuro, hacia el desarrollo de la industria.

Lograr que Ecuador sea reconocido como un país que sabe trabajar, que sabe servir y atender, es decir que sabe hacer las cosas bien. Desarrollar unidos al país que se desea, sonriendo al cliente, sin mentiras y con gestos amable, con parámetros de 
evaluación estrictos, normas de calidad, servicios post-ventas, encuestas de calidad, haciendo de Ecuador profesional en servicio; hacer de Ecuador un país feliz, un país que sonríe, un país innovador, un país de gente que sabe servir.

\section{REFERENCIAS BIBLIOGRÁFICAS}

Blanchard K. (1996) Empowerment. Grupo Editorial Norma.

Bastos A.I (2007). Fidelización al cliente.

Chiavenato, I. (2009). Gestión del talento Humano. Editorial McGraw-Hill.

Giraldo J. C. (2011). Atención al Cliente. Bogotá: Kimpres Ltda.

Koontz, Weihrich y Cannice (2012) Administración, una perspectiva global y empresarial. Editorial McGrawHill.

McGregor D. (1994). El lado humano de las Organizaciones. Editorial McGraw-Hill.

Tigani D. (2006), Excelencia en Servicio.

https://www.gerencie.com/teoria-x-y-teoria-y-de-mcgregor.html

https://www.aiteco.com/teoria-x-y-teoria-y-de-mcgregor/

http://empresarialefectivo.blogspot.com/2013/06/el-servicio-de-calidad-5-estrellas.

html

http://www.eluniversal.com/noticias/opinion/servicio-tiempos-crisis_37184. 